УДК 546.65:544.65

\title{
ВЛИЯНИЕ АЦЕТАТ- И ЦИТРАТ-ИОНОВ НА ПРОТЕКАНИЕ ПРОЦЕССА КАТОДНОГО ВОССТАНОВЛЕНИЯ ҮЬ(ІІІ) НА РТУТНЫХ ЭЛЕКТРОДАХ
}

\author{
Коршунов Андрей Владимирович',
} korshunov@tpu.ru

Ковалева Светлана Владимировна², svetkovaleva@rambler.ru

\author{
Абрамова Полина Владимировна', \\ polya.bozhcko@yandex.ru \\ Горлушко Дмитрий Александрович', \\ gda@tpu.ru \\ 1 Национальный исследовательский Томский политехнический университет, \\ Россия, 634050, г. Томск, пр. Ленина, 30. \\ 2 Томский государственный педагогический университет, \\ Россия, 634061, г. Томск, ул. Киевская, 60.
}

\begin{abstract}
Актуальность. Иттербий, его сплавы и соединения характеризуются особыми термоэлектрическими, магнитными и оптическими свойствами; иттербийсодержащие материалы находят применение в военной и атомной промышленности. Вследствие высокой химической активности, а также сходства с другими редкоземельными элементами технологии выделения иттербия включают большое количество операций, в их числе осаждение, электролиз, цементация, адсорбция и др. Особенностью иттербия (а также европия и самария) по сравнению с другими редкоземельными элементами является возможность катодного осаждения на ртутном электроде и образования амальгамы при сравнительно невысоких отрицательных потенциалах. В связи с этим исследование закономерностей протекания электродных процессов с участием иттербия является актуальной задачей как с фундаментальной, так и с технологической точки зрения.

Цель: определить особенности влияния комплексообразователей (ацетат- и цитрат-ионов) на протекание процесса катодного восстановления Yb(III) на ртутных электродах.

объекты: оксид иттербия, хлорная и уксусная кислоты, растворы солей $\left(\mathrm{NaClO}_{4}, \mathrm{LiCl}, \mathrm{N}\left(\mathrm{C}_{2} \mathrm{H}_{5}\right)_{4} \mathrm{Cl}\right.$, ацетата и цитрата натрия) и щелочей $(\mathrm{NaOH})$.

Методы: полярография постоянного и переменного тока, циклическая вольтамперометрия.

Результаты. Уточнена область потенциалов протекания катодного процесса $Y b(I I I) \rightarrow Y b(I I)\left(12 \cdot 10^{-4} \ldots 1 \cdot 10^{-3} \mathrm{M}\right)$ на ртутных электродах (капающем, стационарном) в некомплексообразующих и комплексообразующих (ацетатном, цитратном) фоновых электролитах в широком интервале $\mathrm{pH}=2 . . .9$. Установлено влияние комплексообразования Yb(III) на соотношение токов восстановления ионов иттербия и водорода и на устойчивость промежуточного продукта Yb(II) катодного процесса в среде раствора. Показан каталитический характер химической реакции окисления Yb(II) ионами водорода в прикатодном слое раствора на протекание процесса $Y b(I I I) \rightarrow Y b(I I)$ в области потенциалов $-1,3 . . .-1,5$ B (нас. х.с.э.). Интерпретация результатов электрохимических исследований проведена с использованием расчётных диаграмм E-pH для систем $\mathrm{Yb}^{-} \mathrm{H}_{2} \mathrm{O}-\mathrm{CH}_{3} \mathrm{COO}^{-}$и Yb- $\mathrm{H}_{2} \mathrm{O}-\mathrm{Cit}^{3-}$ в сравнении с системой без комплексообразователя. Показана важность учёта состояния Yb(III) в растворе и условий электролиза при протекании катодного процесса.
\end{abstract}

\section{Ключевые слова:}

Соединения иттербия (III), ацетатные и цитратные комплексы, постоянно- и переменнотоковая полярография, циклическая вольтамперометрия, ртутные электроды, катодное восстановление.

\section{Введение}

Материалы на основе иттербия и его соединений обладают особыми термоэлектрическими, магнитными и оптическими свойствами и находят применение в военной и атомной промышленности. Вследствие высокой химической активности, а также сходства с другими редкоземельными элементами технологии выделения иттербия включают большое количество операций, в их числе осаждение, электролиз, цементация, адсорбция и др. [1]. Особенностью иттербия (а также европия и самария) по сравнению с другими редкоземельными элементами является возможность катодного осаждения на ртутном электроде и образования амальгамы при сравнительно невысоких отрицательных потенциалах [2]. С этой особенностью иттербия связаны перспективы его отделения от лютеция при производстве радиоактивных препаратов медицинского назначения [3-5]. Несмотря на достаточно продолжительный период исследований эффективности амальгамного разделения иттербия и лютеция, полного понимания происходящих при этом процессов нет до сих пор. В связи с этим изучение процессов катодного восстановления $\mathrm{Yb}(\mathrm{III})$ в растворах является актуальной фундаментальной и прикладной задачей.

Из ранних работ известно $[1,2,6]$, что на ртутном катоде, а также на амальгамах щелочных ме- 
таллов иттербий (III) восстанавливается ступенчато через одноэлектронную $\mathrm{Yb}^{3+} \rightarrow \mathrm{Yb}^{2+}$ и двухэлектронную $\mathrm{Yb}^{2+} \rightarrow \mathrm{Yb}^{0}$ стадии с последующим образованием амальгамы. При этом последовательность восстановления ионов водорода, щелочного и редкоземельного металла зависит от разности потенциалов, рН среды, присутствия комплексообразователя. Наиболее оптимальными условиями извлечения иттербия из растворов с образованием амальгамы являются $\mathrm{pH}<7$, многократное повторение операции цементации в сочетании с электролизом, введение в растворы ацетата и цитрата натрия, охлаждение электролита, дополнительное хроматографическое разделение, при этом степень извлечения может достигать $99 \%$ [4, 6-14]. В ряде работ указано, что вследствие высоких значений соотношения иттербий/лютеций (несколько тысяч) на практике метод цементации может быть малоэффективным за счет снижения выхода лютеция $[4,7,15,16]$.

Электрохимическое поведение иттербия изучено преимущественно с применением ртутных электродов. В отсутствие комплексообразователей в хлорнокислых растворах стадия восстановления $\mathrm{Yb}^{3+} \rightarrow \mathrm{Yb}^{2+}$ сопровождается разрядом водорода в области $-1,45$ В, наибольшее влияние которого наблюдается при $\mathrm{pH}<4$ [17]. В работе [18] показано, что в этих условиях обратимое восстановление иттербия сопровождается гомогенными реакциями и контролируется диффузией. Вторая стадия восстановления $\mathrm{Yb}^{2+} \rightarrow \mathrm{Yb}^{0}$ на капающем ртутном электроде [19] также сопровождается восстановлением водорода, ионы $\mathrm{Yb}(\mathrm{II})$ восстанавливаются до металла, а далее происходит каталитическая реакция взаимодействия металла с протонами или молекулами воды на поверхности электрода. При $\mathrm{pH}>4$ значительно усиливаются гидролитические процессы. Потенциал полуволны восстановления $\mathrm{Yb}^{3+}$ до $\mathrm{Yb}^{2+}$ варьируется от 1,46 до 1,40 в зависимости от $\mathrm{pH}$ раствора, потенциал второй ступени восстановления $\mathrm{Yb}^{2+}$ до $\mathrm{Yb}^{0}$ принимает значения от 2,07 до 2,00 В [20].

Анализ опубликованных данных показал, что, несмотря на большое число работ, посвященных исследованию процессов катодного восстановления и амальгамной цементации иттербия из водных растворов, данные по электрохимическому поведению иттербия в растворах ацетатов и цитратов в проанализированных источниках отсутствуют. В связи с этим целью настоящей работы являлось определение особенностей влияния комплексообразователей (ацетат- и цитрат-ионов) на протекание процесса катодного восстановления $\mathrm{Yb}(\mathrm{III})$ на ртутных электродах.

\section{Материалы и методы исследования}

В качестве исходного реагента для приготовления растворов использовали оксид иттербия $(99,9$ мас. \%), предварительно прокаленный при $800{ }^{\circ} \mathrm{C}(4$ ч). Навеску оксида растворяли в 1-2 мл концентрированной хлорной кислоты («х.ч.»), да- лее доводили до заданного объема раствора бидистилированной водой. Концентрация исходного раствора составляла $0,01 \mathrm{M}, \mathrm{pH} \approx 2$. Рабочие растворы готовили путем разбавления исходного бидистиллированной водой, $\mathrm{pH}$ растворов регулировали в интервале $2 . . .9$ путем добавления $\mathrm{HClO}_{4}$ или $\mathrm{NaOH}$. Измерение $\mathrm{pH}$ проводили при помощи $\mathrm{pH}-$ метра pH-410 (г. Гомель, Белоруссия) с универсальным электродом, калибровку рН-метра производили с использованием стандартных буферных растворов с рН 1,65, 4,01 и 6,86.

Электродные процессы с участием иттербия в растворах изучали с использованием классической и переменнотоковой полярографии (полярограф ПУ-1) с ртутным капающим электродом и циклической вольтамперометрии (потенциостат ПИ-50-1.1) со стационарным электродом «ртутная капля» (ртуть Р-0). В работе использовали трехэлектродную ячейку, вспомогательным электродом являлся платиновый электрод, электродом сравнения служил насыщенный хлорсеребряный электрод (нас. х.с.э.). Значения потенциалов приведены относительного этого электрода. В качестве фоновых электролитов использовали $0,1 \mathrm{M}$ растворы $\mathrm{NaClO}_{4}, \mathrm{LiCl}, \mathrm{N}\left(\mathrm{C}_{2} \mathrm{H}_{5}\right)_{4} \mathrm{Cl}$, ацетата и цитрата натрия. Чистота реагентов соответствовала квалификации «х.ч.», дополнительную их очистку не производили. Непосредственно перед электрохимическими измерениями исследуемые растворы деаэрировали путем пропускания азота высокой чистоты.

\section{Результаты и их обсуждение}

В условиях полярографии с капающим электродом катодный процесс $\mathrm{Yb}^{3+}+\mathrm{e}=\mathrm{Yb}^{2+}$ как в некомплексообразующих растворах $\left(\mathrm{NaClO}_{1}, \mathrm{LiCl}\right)$, так и в растворах ацетата натрия отображается на полярограммах в виде одной выраженной необратимой волны (рис. 1). В области $\mathrm{pH}<4$ на восстановление ионов $\mathrm{Yb}^{3+}\left(1 \cdot 10^{-4} \ldots 1 \cdot 10^{-3} \mathrm{M}\right)$ накладывается процесс восстановления ионов водорода, что приводит к существенному возрастанию предельного тока полярографической волны. При этом волны иттербия и водорода плохо разделяются, этот эффект также наблюдается на переменнотоковых полярограммах в виде раздвоенного максимума. В интервале $4<\mathrm{pH}<6$ волна восстановления водорода смещается в область отрицательных потенциалов и её предельный ток значительно снижается, что позволяет выделить волну восстановления ионов иттербия $\left(E_{1 / 2}=-1,30 \ldots-1,38\right.$ В). В этой области $\mathrm{pH}$ величина предельного тока пропорциональна концентрации $\mathrm{Yb}^{3+}$ в растворе. При $\mathrm{pH} \geq 6,25$ в некомплексообразующей среде наблюдается осаждение нерастворимого гидроксида $\mathrm{Yb}(\mathrm{OH})_{3}$, при этом волна восстановления ионов иттербия на полярограммах не проявляется. Это свидетельствует о том, что в условиях эксперимента гидроксид иттербия электрохимически неактивен и не подвергается катодному восстановлению.

В фоновых растворах ацетата натрия полярографические волны иттербия и водорода разделяются 

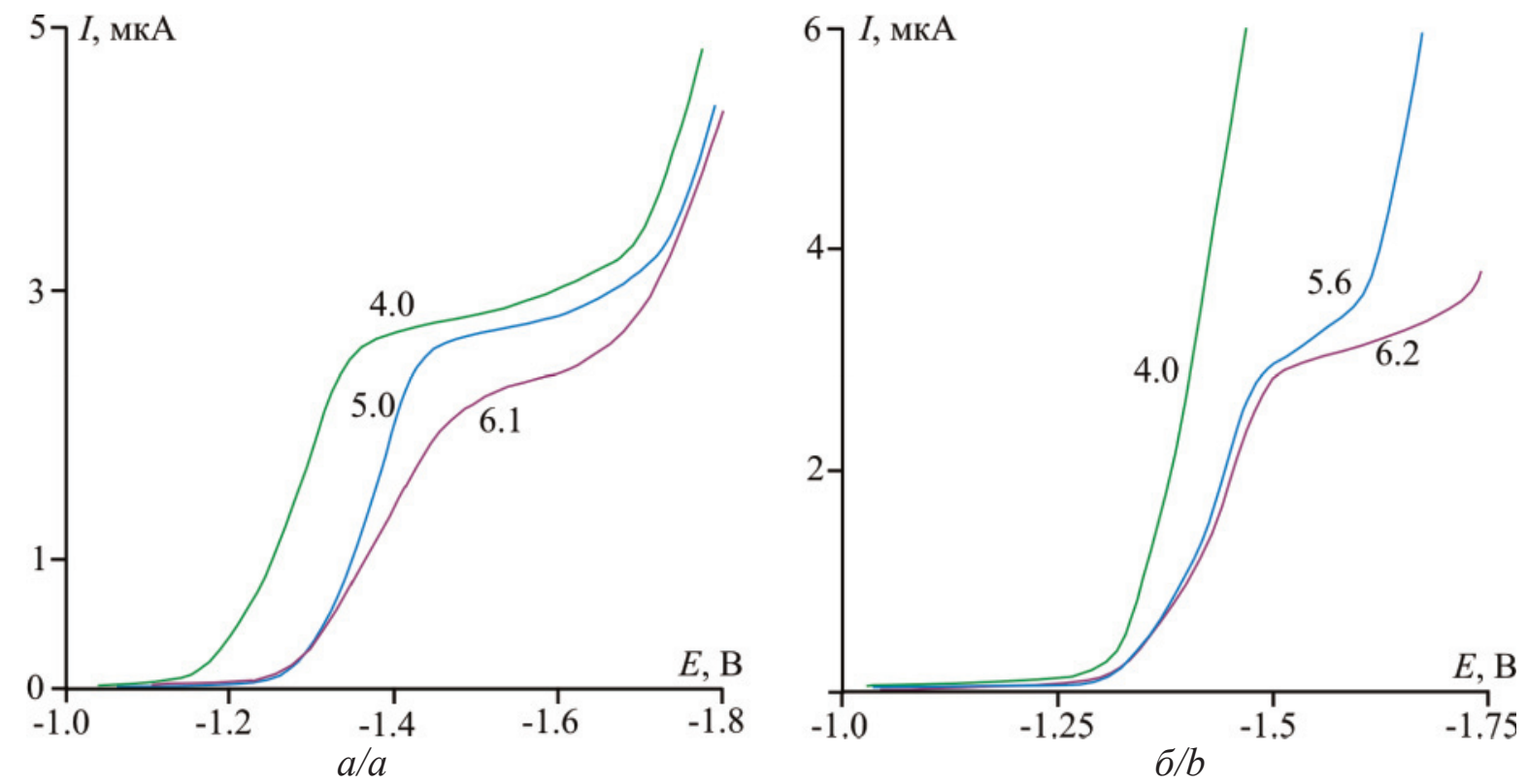

Puc. 1. Постояннотоковые полярограмлы восстановления $Y b(I I I)\left(5 \cdot 10^{-4} \mathrm{M}\right)$ на ртутном капающем электроде в (а) перхлоратном $\left(0,1 \mathrm{M} \mathrm{NaClO}_{4}\right)$ и (б) ацетатном (0,1 $\mathrm{M} \mathrm{CH}_{3} \mathrm{COONa)}$ фоновых электролитах при различных значениях рН растворов (значения рН указаны на рис.; паралетры капающего электрода: скорость истечения $\mathrm{Hg}$ 4,6 мг/с, период капания 5,7 с; скорость развёртки потенииалов $3 \mathrm{mB} / \mathrm{c}$ )

Fig. 1. Direct-current polarograms of $\mathrm{Yb}(\mathrm{III})$ reduction $\left(5 \cdot 10^{-4} \mathrm{M}\right)$ at dropping $\mathrm{Hg}$-electrode in (a) perchlorate $\left(0,1 \mathrm{M} \mathrm{NaClO}{ }_{4}\right)$ and $(\mathrm{b})$ acetate $\left(0,1 \mathrm{M} \mathrm{CH}_{3} \mathrm{COONa}\right.$ ) supporting electrolytes at different $\mathrm{pH}$ of the solutions (the values of $\mathrm{pH}$ are shown in the Fig.; $\mathrm{Hg}$ flow rate is $4,6 \mathrm{mg} / \mathrm{s}$; dropping period is $5,7 \mathrm{~s}$; potential sweep rate is $3 \mathrm{mV} / \mathrm{s}$ )

хуже, чем в перхлоратных растворах (рис. 1, б). Наложение двух катодных процессов в этом случае проявляется до более высоких значений рН. При этом область выделения водорода смещается в направлении более положительных потенциалов, что свидетельствует об электрокаталитическом влиянии комплексов $\mathrm{Yb}$. За счет формирования комплексов наблюдается смещение потенциала полуволны восстановления ионов $\mathrm{Yb}^{3+}$ в область отрицательных потенциалов, $E_{1 / 2}=-1,42 . .-1,45$ В. Величины предельных токов восстановления $\mathrm{Yb}(\mathrm{III})$ при $\mathrm{pH}=5 . .6$ в перхлоратных и ацетатных растворах сопоставимы между собой (рис. $1, a$, б). Несмотря на образование комплексов $\mathrm{Yb}$ в ацетатных фоновых электролитах, повышение $\mathrm{pH}$ среды до 6,5 и более приводит к осаждению иттербия из раствора в виде гидроксида и к исчезновению волны восстановления иттербия на полярограммах. Следовательно, использование ацетатных растворов позволяет лишь незначительно повысить значения $\mathrm{pH}$ растворов, при которых происходит восстановление $\mathrm{Yb}^{3+}$. При этом существенного снижения влияния разряда водорода на исследуемый процесс практически не происходит. Нужно отметить, что вторая волна, соответствующая процессу $\mathrm{Yb}^{2+}+2 \mathrm{e}=\mathrm{Yb}$, на полярограммах не была зафиксирована.

В отличие от перхлоратных и ацетатных фоновых растворов, в среде $0,1 \mathrm{M}$ цитрата натрия в исследованном интервале $\mathrm{pH}$ волна восстановления ионов иттербия на полярограммах не проявляется. По-видимому, цитратный комплекс [Yb(Cit)], образующийся при большом избытке цитрат-ионов в растворе, не подвергается катодному восстановлению в условиях полярографии. При соотношениях концентраций цитрат натрия $/ \mathrm{Yb}^{3+}=1 . .3$ в раствоpe $0,1 \mathrm{M} \mathrm{NaClO}_{4}$ происходит значительное смещение $E_{1 / 2}$ волны от $-1,38$ до $-1,65$ В, при этом предельный ток снижается и достигает фоновых значений при дальнейшем увеличении концентрации цитрат-ионов в смеси. Нужно отметить, что повышение $\mathrm{pH}$ цитратных растворов до 10 не приводило к осаждению гидроксида иттербия вследствие высокой прочности комплекса, ток восстановления цитратного комплекса иттербия в щелочной среде также не наблюдается.

Более детальное изучение особенностей протекания электродных процессов проведено с использованием циклической вольтамперометрии со стационарной $\mathrm{Hg}$-каплей в качестве рабочего электрода. Из анализа вольтамперограмм следует, что в некомплексообразующих и комплексообразующих фоновых электролитах электродные процессы с участием $\mathrm{Yb}(\mathrm{III})$ в значительной степени различаются (рис. 2).

В растворах $0,1 \mathrm{M} \mathrm{NaClO}, \mathrm{N}\left(\mathrm{C}_{2} \mathrm{H}_{5}\right)_{4} \mathrm{Cl}, \mathrm{KCl}$ на катодном участке ЦВА проявляется максимум тока процесса восстановления $\mathrm{Yb}^{3+}+\mathrm{e}=\mathrm{Yb}^{2+}$ при $E_{\max }=-1,4 \ldots-1,5 \mathrm{~B}$, величина которого пропорциональна концентрации ионов $\mathrm{Yb}^{3+}$ в растворе. При дальнейшем смещении потенциала электрода вплоть до -2 В происходит возрастание тока катодного восстановления водорода, дополнительных максимумов, связанных с более глубоким восстановлением иттербия, не наблюдается. При обратной 


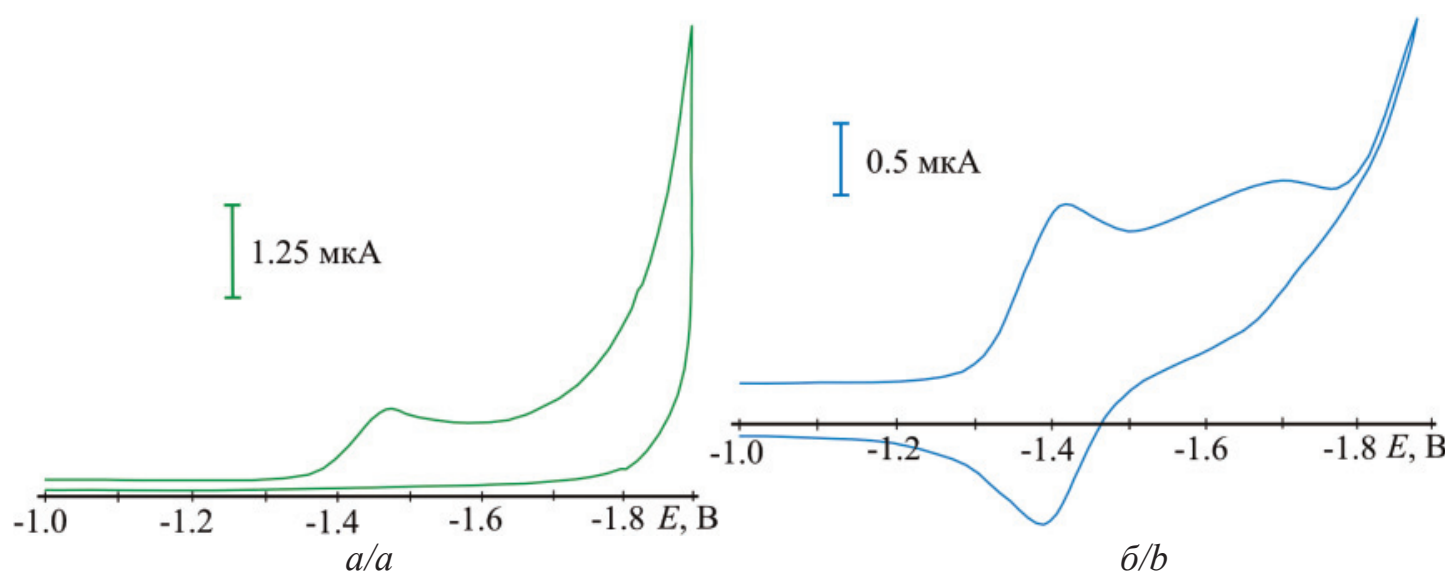

Puс. 2. Циклические вольтамперограммы стационарного Нg-электрода в растворах $Y b(I I I)\left(5 \cdot 10^{-4} \mathrm{M}\right)$ на фоне (а) перхлората натрия $\left(0,1 \mathrm{M} \mathrm{NaClO}_{4}\right)$ и (б) ацетата натрия $\left(0,1 \mathrm{M} \mathrm{CH}_{3} \mathrm{COONa}\right.$ ) при $\mathrm{pH}=5$ (скорость развертки потенциалов $50 \mathrm{mB} / \mathrm{c}$ )

Fig. 2. Cyclic voltammograms of the hanging mercury drop electrode in $\mathrm{Yb}$ (III) solutions $\left(5 \cdot 10^{-4} \mathrm{M}\right)$ in (a) sodium perchlorate $\left(0,1 \mathrm{M} \mathrm{NaClO}_{4}\right)$ and in (b) sodium acetate $\left(0,1 \mathrm{M} \mathrm{CH}_{3} \mathrm{COONa}\right.$ ) at $\mathrm{pH}=5$ ( sweep rate is $50 \mathrm{mV} / \mathrm{s}$ )

развертке потенциалов на анодном участке ЦВА максимумы также отсутствуют. Следовательно, процесс $\mathrm{Yb}^{3+}+\mathrm{e}=\mathrm{Yb}^{2+}$ в указанных электролитах протекает необратимо, продукт восстановления $\left(\mathrm{Yb}^{2+}\right)$ является неустойчивым и не накапливается в приэлектродном растворе. Очевидно, что ионы $\mathrm{Yb}^{2+}$ за счет значительного отрицательного стандартного окислительно-восстановительного потенциала $E^{\circ}\left(\mathrm{Yb}^{3+} / \mathrm{Yb}^{2+}\right)=-1,05$ В (ст.в.э.) непосредственно после образования вступают в химическую реакцию с катионами водорода $\mathrm{Yb}^{2+}+\mathrm{H}^{+}=\mathrm{Yb}^{3+}+1 / 2 \mathrm{H}_{2}$. Протекание этой реакции обусловливает каталитическое увеличение значения $I_{\max }$ и позволяет объяснить отсутствие соответствующего максимума тока окисления катиона $\mathrm{Yb}^{2+}$ на анодном участке ЦВА. Кроме того, расходование катионов $\mathrm{Yb}^{2+}$ при протекании химической стадии приводит к невозможности более глубокого восстановления с образованием амальгамы, что подтверждается отсутствием максимумов токов на ЦВА в области отрицательных потенциалов. Следовательно, в некомплексообразующих фоновых электролитах протекание катодного процесса с участием $\mathrm{Yb}$ (III) фактически не приводит к накоплению продуктов восстановления вследствие их расходования в каталитической химической стадии.

В условиях ЦВА в фоновом электролите $0,1 \mathrm{M}$ $\mathrm{CH}_{3} \mathrm{COONa}$ катодный и анодный участки вольтамперограмм содержат по два максимума токов (рис. 2, б). Катодные максимумы при $-1,45$ и -1,7 В отображают протекание электродной реакции $\mathrm{Yb}(\mathrm{III}) \rightarrow \mathrm{Yb}(\mathrm{II})$ восстановления ацетатных комплексов различного состава. При обратной развертке потенциалов на анодном участке ЦВА отображаются соответствующие процессы окисления. Вместе с тем сопоставление этих максимумов показало, что величины катодных токов не согласуются между собой, как и соответствующие им анодные токи. Величина первого катодного тока $I_{\max , 1}$ при $E=-1,45$ В превышает величину токов остальных максимумов вследствие наложения ка- талитической химической стадии с участием $\mathrm{Yb}(\mathrm{II})$ и ионов водорода. Величина второго катодного тока $I_{\max , 2}$ значительно меньше величины первого вследствие частичного окисления $\mathrm{Yb}(\mathrm{II})$ в растворе. Тем не менее, в отличие от некомплексообразующих фоновых растворов, в растворе ацетата натрия происходит стабилизация ионов Yb(II) за счет образования ацетатных комплексов, которые накапливаются в приэлектродном слое раствора. В связи со стабилизацией $\mathrm{Yb}(\mathrm{II})$ на анодном участке ЦВА отображается максимум анодного тока окисления этих ионов, чего не наблюдалось в случае перхлоратных и хлоридных сред. Тем не менее, величины токов окисления промежутоных продуктов восстановления существенно меньше токов катодного процесса, что свидетельствует 0 каталитическом влиянии химической реакции на протекание катодного процесса.

С целью обоснования выводов об особенностях протекания электродных процессов в некомплексоообразующих и комплексообразующих фоновых растворах были проведены расчёты диаграмм $E-\mathrm{pH}$ для исследуемых систем (рис. 3). В области потенциалов устойчивости воды термодинамически устойчивым состоянием иттербия является $\mathrm{Yb}(\mathrm{III})$ (рис. $3, a)$, которое в зависимости от $\mathrm{pH}$ раствора представлено катионами или гидроксидом иттербия. Восстановление Yb(III) термодинамически возможно при крайне отрицательных потенциалах за пределами устойчивости воды, при этом катион $\mathrm{Yb}^{2+}$ должен окисляться водой с выделением водорода (рис. $3, a)$. Действительно, потенциал катодного процесса $\mathrm{Yb}(\mathrm{III}) \rightarrow \mathrm{Yb}(\mathrm{II})$, наблюдаемый в эксперименте (рис. 1,2$)$, близок к величине стандартного потенциала $-1,05$ В (ст.в.э.) (рис. $3, a)$, процесс протекает благодаря высокому перенапряжению водорода на ртутном электроде. Окисление $\mathrm{Yb}^{2+}$ в ходе химической реакции, протекающей в приэлектродном слое раствора, хорошо согласуется с областью потенциалов устойчивости этого катиона на диаграмме $E-\mathrm{pH}$. 


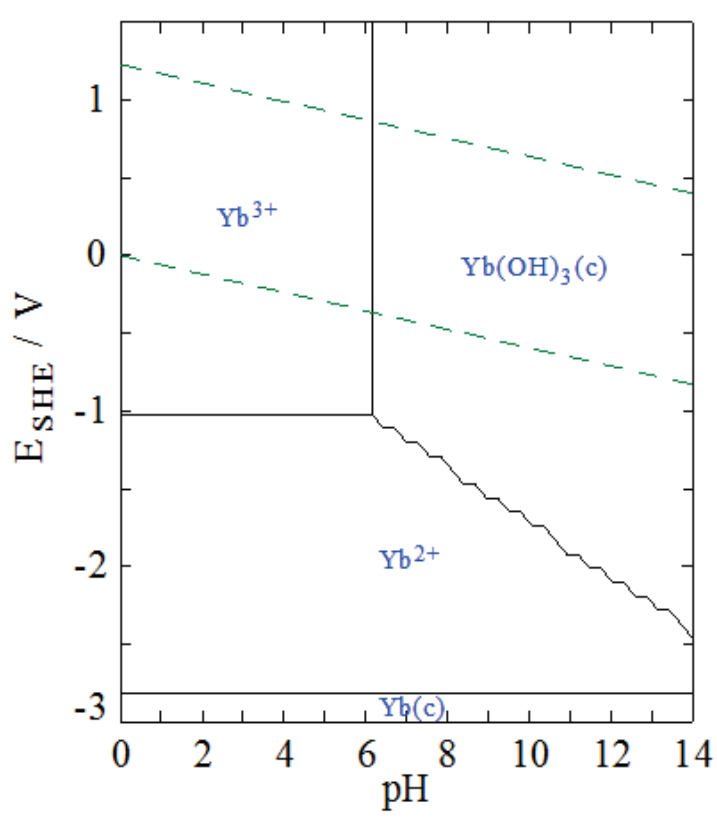

$a / a$

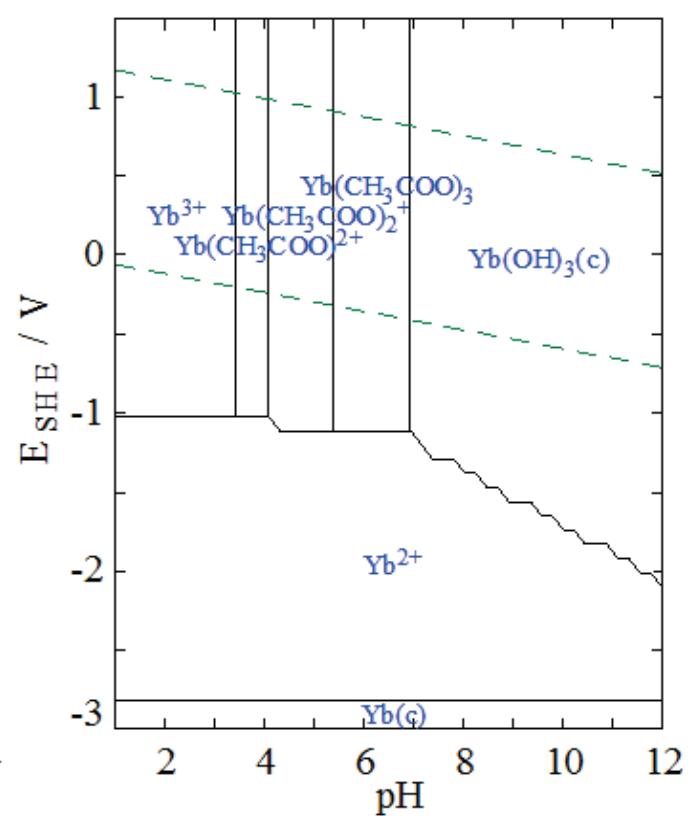

$\sigma / b$

Puc. 3. Расчётные диаграмлы $E_{\text {(ст.в.). }}-p H$ для систем (а) $\mathrm{Yb}-\mathrm{H}_{2} \mathrm{O}$ u (б) $\mathrm{Yb}-\mathrm{H}_{2} \mathrm{O}-\mathrm{CH}_{3} \mathrm{COO}^{-}\left(c_{0}\left(\mathrm{Yb}^{3+}\right)=5 \cdot 10^{-4} \mathrm{M} ; c_{0}\left(\mathrm{CH}_{3} \mathrm{COO}^{-}\right)=0,1 \mathrm{M} ; 25^{\circ} \mathrm{C}\right)$

Fig. 3. Potential-pH diagrams for the systems ( a ) $\mathrm{Yb}-\mathrm{H}_{2} \mathrm{O}$ and (b) $\mathrm{Yb}-\mathrm{H}_{2} \mathrm{O}-\mathrm{CH}_{3} \mathrm{COO}^{-}\left(c_{\mathrm{o}}\left(\mathrm{Yb}^{3+}\right)=5 \cdot 10^{-4} \mathrm{M} ; \mathrm{c}_{\mathrm{o}}\left(\mathrm{CH}_{3} \mathrm{COO}^{-}\right)=0,1 \mathrm{M} ; 25^{\circ} \mathrm{C}\right)$

В ацетатном фоновом электролите иттербий образует несколько комплексных соединений с различным числом лигандов и различной устойчивостью (рис. 3, б). Несмотря на формирование комплексов в системе области потенциалов и $\mathrm{pH}$ устойчивости катиона $\mathrm{Yb}^{2+}$ и гидроксида иттербия изменяются незначительно, что хорошо согласуется с данными полярографии и вольтамперометрии (рис. 1,2 ). Детальный анализ диаграммы (рис. 3, б) позволяет определить смещение потенциала восстановления $\mathrm{Yb}(\mathrm{III}) \rightarrow \mathrm{Yb}(\mathrm{II})$ для комплексов различного состава: катионный комплекс с одним лигандом $\left[\mathrm{Yb}\left(\mathrm{CH}_{3} \mathrm{COO}\right)\right]^{2+}$ характеризуется таким же потенциалом восстановления, как и катион $\mathrm{Yb}^{3+}$ $(-1,05$ В), тогда как для комплексов с бо́льшим числом лигандов $\left[\mathrm{Yb}\left(\mathrm{CH}_{3} \mathrm{COO}\right)_{2}\right]^{+}$и $\left[\mathrm{Yb}\left(\mathrm{CH}_{3} \mathrm{COO}\right)_{3}\right]$ характерно смещение потенциала восстановления на $\sim 0,2$ В в сторону более отрицательных потенциалов.

Этот факт позволяет объяснить появление второго широкого максимума катодного тока на ЦВА для ацетатных растворов (рис. 2, б). По-видимому, катионы $\mathrm{Yb}^{2+}$, образующиеся при восстановлении комплексов $\mathrm{Yb}(\mathrm{III})$, образуют с ацетат-ионами комплексы аналогичного состава, что согласуется с положением максимумов анодного тока на ЦВА (рис. 2, б).

В отличие от ацетатного фонового электролита, с цитрат-ионами $\mathrm{Yb}(\mathrm{III})$ образует один нейтральный комплекс [Yb(Cit)], устойчивый в более широких интервалах $\mathrm{pH}$ и потенциалов (рис. 4). Электродные процессы с участием иттербия в избытке цитрата в области потенциалов $-1,3 . . .-1,5$ В, как это наблюдается для перхлоратных и ацетатных растворов, не протекают. Такая особенность, повидимому, связана с низким значением константы нестойкости, отсутствием заряда у комплекса, структурой органических лигандов. В связи с этим технологически значимым фактором в процессах электролитического и амальгамного извлечения иттербия из растворов с применением цитратов является точный контроль содержания цитрат-ионов в растворах.

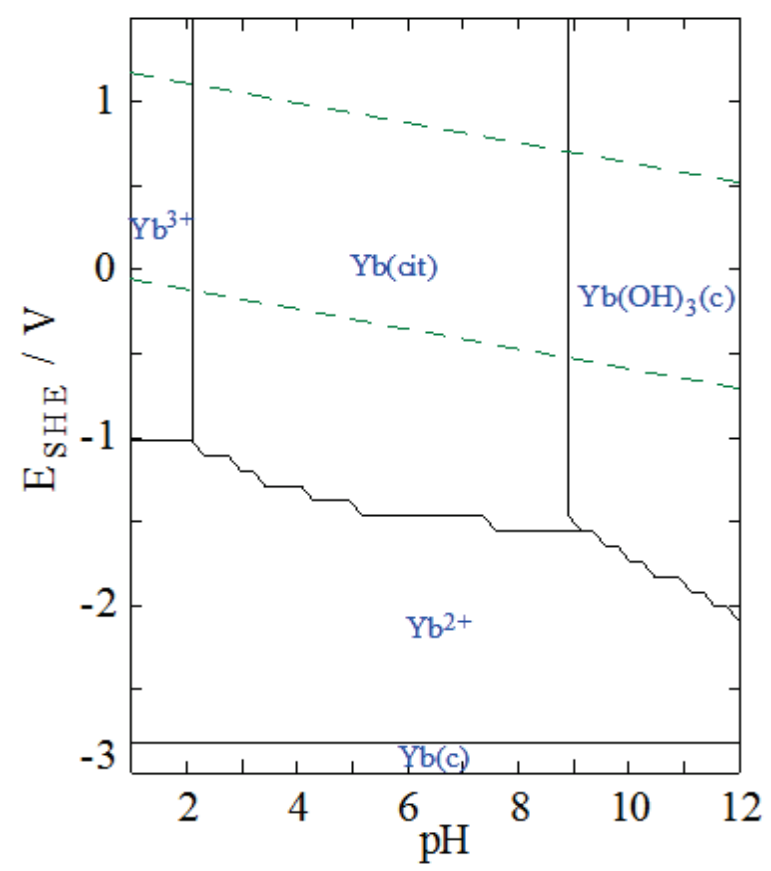

Pис.4. Расчётная диаграмла $E_{\text {(ст.в.э) }}-p H \quad$ для системь $\mathrm{Yb}-\mathrm{H}_{2} \mathrm{O}-\mathrm{Cit}^{3-}\left(c_{\mathrm{o}}\left(\mathrm{Yb}^{3+}\right)=5 \cdot 10^{-4} \mathrm{M} ; c_{\mathrm{o}}\left(\mathrm{Cit}^{3-}\right)=0,1 \mathrm{M} ; 25^{\circ} \mathrm{C}\right)$

Fig. 4. Potential-pH diagram for the system $\mathrm{Yb}^{-} \mathrm{H}_{2} \mathrm{O}-\mathrm{Cit}^{3-}$ $\left(c_{0}\left(\mathrm{Yb}^{3+}\right)=5 \cdot 10^{-4} \mathrm{M} ; \mathrm{c}_{0}\left(\mathrm{Cit}^{3-}\right)=0,1 \mathrm{M} ; 25^{\circ} \mathrm{C}\right)$ 


\section{Выводы}

1. Параметры первой стадии восстановления иттербия(III) $\mathrm{Yb}(\mathrm{III}) \rightarrow \mathrm{Yb}(\mathrm{II})$ на ртутном катоде (потенциал, ток), а также устойчивость продукта $\mathrm{Yb}(\mathrm{II})$ восстановления определяются присутствием комплексообразователей в растворе. Ионы $\mathrm{Yb}^{2+}$ в приэлектродном растворе быстро окисляются ионами водорода (водой), химическая стадия оказывает каталитический эффект на величину катодного тока восстановления Yb(III). В присутствии ацетат-ионов происходит стабилизация Yb(II), его образование в растворе может быть определено по максимуму тока анодного оки-

\section{СПИСОК ЛИТЕРАТУРЫ}

1. Рябчиков Д.И., Терентьева Е.А. Новое в способах разделения редкоземельных элементов // Успехи химии. - 1960. - Т. 2. № 10. - C. $1285-1300$.

2. Колесов Г.М., Панкратова Л.Н. Разделение редкоземельных элементов электролизом на ртутном катоде // Успехи химии. 1968. - T. 37. - № 9. - C. 1642-1657.

3. Update on radionuclide therapy in oncology (Review) / C. Nitipir, D. Niculae, C. Orlov, M. Barbu et al. // Oncology Letters. 2017. - V. 14. - № 6. - P. 7011-7015.

4. Comparative studies of extraction chromatography and electroamalgamation separation to produce no-carrier added ${ }^{177} \mathrm{Lu}$ by Tehran research reactor / N. Salek, M. Shamsaei, M. Maragheh, S. Arani, A. Samani // Iran J. Nucl. Med. - 2017. - V. 25. № 1. - P. 23-33.

5. Тарасов В.А., Романов Е.Г., Кузнецов Р.А. Сравнительный анализ схем реакторной наработки лютеция-177 // Известия самарского научного центра российской академии наук. 2013. - T. 15. - № 4 (5). - C. 1084-1090.

6. Marsh J.K. Rare-earth metal amalgams. P. III. The separation of ytterbium from its neighbours // J. Chem. Soc. - 1943. - P. 8-10.

7. Cieszykowska I., Zoltowska M., Mielcarski M. Separation of ytterbium from ${ }^{177} \mathrm{Lu} / \mathrm{Yb}$ mixture by electrolytic reduction and amalgamation // Sop Transactions on Applied Chemistry. 2014. - V. 1. - № 2. - P. 6-13.

8. An electro-amalgamation approach to isolate no-carrier-added ${ }^{17} \mathrm{Lu}$ from neutron irradiated $\mathrm{Yb}$ for biomedical applications / R. Chakravarty, T. Das, A. Dash, M. Venkatesh // Nuclear Medicine and Biology. - 2010. - V. 37. - P. 811-820.

9. Electrochemical method for producing radionuclide $\mathrm{Lu}-177$ with high specific activity / P.P. Boldyrev, A.V. Kurochkin, R.F. Nurtdinov, M.A. Proshin, D.Yu. Chuvilin, Yu.A. Yashin // Moscow Univ. Chem. Bull. - 2016. - V. 71. - № 3. - P. 193-198.

10. Moeller T., Kremers H. Observations on the rare earths extraction of ytterbium from rare earth mixtures with sodium amalgam // Ind. Eng. Chem. - 1945. - V. 17. - № 12. - P. 798-800. сления в условиях циклической вольтамперометрии.

2. Избыточная концентрация цитрат-ионов по отношению к $\mathrm{Yb}(\mathrm{III})$ в растворе приводит к образованию прочного комплекса, который, как и гидроксид иттербия, в условиях циклической вольтамперометрии не восстанавливается на $\mathrm{Hg}$ катоде вплоть до крайне отрицательных потенциалов. Для процессов электролитического и цементационного извлечения иттербия из растворов является нецелесообразным применение цитратных фоновых растворов, а также создание $\mathrm{pH}>6,2$ вследствие потерь иттербия за счет осаждения в виде малорастворимых соединений.

11. Possibility of obtaining high-activity ${ }^{177} \mathrm{Lu}$ in the IR-8 research reactor / P.P. Boldyrev, V.A. Zagryadskii, D.Yu. Erak et al. // Atomic Energy. - 2017. - V. 121. - № 3. - P. 208-213.

12. Radiochemical separation of no-carrier-added ${ }^{177} \mathrm{Lu}$ as produced via the ${ }^{176} \mathrm{Yb}(n, \gamma)^{177} \mathrm{Yb} \rightarrow{ }^{177} \mathrm{Lu}$ process / N.A. Lebedev, A.F. Novgorodov, R. Misiak, J. Brockmann, F. Rosch // Applied Radiation and Isotopes. - 2000. - V. 53. - P. 421-425.

13. McCoy H.N. Europium and ytterbium amalgams // J. Am. Chem. Soc. -2002 . - V. 63. - № 6. - P. 1622-1624.

14. Kosynkin V.D., Molchanova T.V., Zharova E.V. Lutetium and ytterbium separation by ion-exchange chromatography // Atomic Energy. - 2017. - V. 121. - № 6. - P. 443-447.

15. Bilewicz A., Zuchowska K., Bartos B. Separation of $\mathrm{Yb}$ as $\mathrm{YbSO}_{4}$ from the ${ }^{176} \mathrm{Yb}$ target for production of ${ }^{177} \mathrm{Lu}$ via the ${ }^{176} \mathrm{Yb}(\mathrm{n}, \gamma)^{177} \mathrm{Yb} \rightarrow{ }^{177} \mathrm{Lu}$ process // Journal of Radioanalytical and Nuclear Chemistry. - 2009. - V. 280. - № 1. - P. 167-169.

16. Indirect production of no carrier added (NCA) ${ }^{177} \mathrm{Lu}$ from irradiation of enriched ${ }^{176} \mathrm{Yb}$ : options for ytterbium/lutetium separation / A. Dash, R. Chakravarty, F.F. Knapp, A. Pillai // Current Radiopharmaceuticals. - 2015. - V. 8. - № 2. - P. 107-118.

17. Steeman E., Temmerman E., Verbeek F. Electrochemical reduction of the lanthanide ions. Part 1. First reduction step of ytterbium (III) in acidic $1 \mathrm{M} \mathrm{NaClO}_{4}$ solution // J. Electroanal. Chem. - 1978. - V. 89. - P. 97-111.

18. Rabockai T. Electrochemical reduction of ytterbium in perchloric media // J. Electroanal. Chem. - 1977. - V. 76. - P. 83-89.

19. Steeman E., Temmerman E., Verbeek F. Electrochemical reduction of the lanthanide ions. Part II. Second reduction step of europium (III), ytterbium (III) and samarium (III) in acidic tetramethylammonium perchlorate solution // J. Electroanal. Chem. 1978. - V. 89. - P. 113-122.

20. Barton G.B., Kurbatov J.D. Dependence of the diffusion current of ytterbium on supporting electrolyte and $\mathrm{pH} / / \mathrm{J}$. Phys. Chem. - 1949. - V. 53. - № 5. - P. 683-690.

Поступила 08.05.2019 2.

\section{Информация об авторах}

Коршунов А.B., доктор химических наук, профессор отделения естественных наук Школы базовой инженерной подготовки Национального исследовательского Томского политехнического университета.

Ковалева C.B., доктор химических наук, профессор кафедры химии и методики обучения химии биолого-химического факультета Томского государственного педагогического университета.

Абрамова П.В., кандидат химических наук, ассистент отделения естественных наук Школы базовой инженерной подготовки Национального исследовательского Томского политехнического университета.

Горлушко Д.А., кандидат химических наук, доцент Научно-образовательного центра Н.М. Кижнера Национального исследовательского Томского политехнического университета. 


\title{
INFLUENCE OF ACETATE AND CITRATE IONS ON CATHODIC REDUCTION OF Yb(III) AT MERCURY ELECTRODES
}

\author{
Andrey V. Korshunov', \\ korshunov@tpu.ru \\ Svetlana V. Kovaleva², \\ svetkovaleva@rambler.ru \\ Polina V. Abramova', \\ polya.bozhcko@yandex.ru

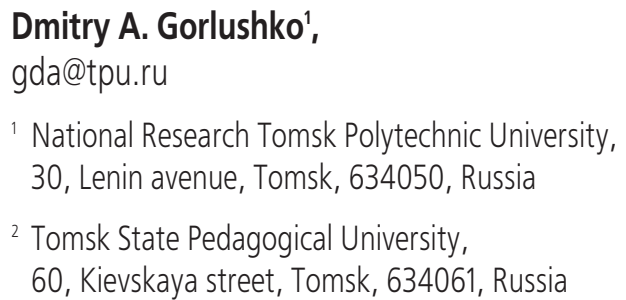

Relevance. Ytterbium, its alloys and compounds are characterized by valuable thermoelectric, magnetic and optical properties, ytterbium-containing materials are used in military and nuclear industries. Due to the high chemical activity, as well as similarities with other rare earth elements, ytterbium isolation technologies include a large number of operations, among them deposition, electrolysis, cementation, adsorption, etc. The peculiarity of ytterbium (as well as europium and samarium) in comparison with other rare earth elements is the possibility of cathodic deposition at the mercury electrode and formation of amalgam at relatively low negative potentials. In this regard, the study of the regularities of the electrode processes involving ytterbium is an urgent task both from a fundamental and technological point of view.

The aim of the research is to determine the features of the effect of complexing agents (acetate and citrate ions) on cathodic reduction of Yb(III) at mercury electrodes.

Objects: ytterbium oxide, perchloric and acetic acids, solutions of salts $\left(\mathrm{NaClO}_{4}, \mathrm{LiCl}, \mathrm{N}\left(\mathrm{C}_{2} \mathrm{H}_{5}\right)_{4} \mathrm{Cl}\right.$, sodium acetate and citrate) and alkali $(\mathrm{NaOH})$.

Methods: direct and alternating current polarography, cyclic voltammetry with mercury electrodes.

Results. The range of potential of the cathodic process $Y b(I I I) \rightarrow Y b(I I)\left(1 \cdot 10^{-4} \ldots 1 \cdot 10^{-3} \mathrm{M}\right)$ at mercury electrodes (dropping, stationary) in non-complexing and complexing (acetate, citrate) supporting electrolytes in a wide $\mathrm{pH}$ range 2...9 has been refined. The effect of complexation of $Y b(I I I)$ on the ratio of ytterbium and hydrogen ions reduction currents and on the stability of the intermediate product $Y b$ (II) of the cathode process in the solution medium was established. The paper introduces the catalytic nature of the chemical reaction of $\mathrm{Yb}$ (II) oxidation by hydrogen ions occurred in the near-cathode layer of the solution to the process $Y b(I I I) \rightarrow Y b(I I)$ at potentials $-1,3 \ldots-1,5 \mathrm{~V}$ ( vs. Ag/AgCl/KCl). Interpretation of the results of electrochemical studies was carried out using the calculated diagrams $\mathrm{E}-\mathrm{pH}$ for $\mathrm{Yb}^{-} \mathrm{H}_{2} \mathrm{O}-\mathrm{CH}_{3} \mathrm{COO}^{-}$and $\mathrm{Yb}^{-} \mathrm{H}_{2} \mathrm{O}-\mathrm{Cit}^{3-}$ systems in comparison with the system without complexing agent. The importance of taking into account $\mathrm{Yb}$ (III) state in solutions and electrolysis conditions for the cathode process is discussed.

\section{Key words:}

Ytterbium(III) compounds, acetate and citrate complexes, direct and alternating current polarography,

cyclic voltammetry, mercury electrodes, cathodic reduction.

\section{REFERENCES}

1. Ryabchikov D.I., Terent'eva E.A. Progress in methods for the separation of rare-earth elements. Russ. Chem. Rev., 1960, vol. 29, no. 10, pp. 589-598.

2. Kolesov G.M., Pankratova L.N. Separation of rare-earth elements by electrolysis at a mercury cathode. Russ. Chem. Rev., 1968, vol. 37, no. 9, pp. 701-710.

3. Nitipir C., Niculae D., Orlov C., Barbu M., et al. Update on radionuclide therapy in oncology (Review). Oncology Letters, 2017, vol. 14, no. 6, pp. 7011-7015.

4. Salek N., Shamsaei M., Maragheh M., Arani S., Samani A. Comparative studies of extraction chromatography and electroamalgamation separation to produce no-carrier added ${ }^{177} \mathrm{Lu}$ by Tehran research reactor. Iran J. Nucl. Med., 2017, vol. 25, no. 1, pp. 23-33.

5. Tarasov V.A., Romanov E.G., Kuznetsov R.A. Sravnitelny analiz skhem reaktornoy narabotki lyutetsiya-177 [Comparative analy- sis of the reactor production of lutetium-177]. Izvestiya Samarskogo nauchnogo tsentra RAS, 2013, vol. 15, no. 4 (5), pp. 1084-1090.

6. Marsh J.K. Rare-earth metal amalgams. P. III. The separation of ytterbium from its neighbours. J. Chem. Soc., 1943, pp. 8-10.

7. Cieszykowska I., Zoltowska M., Mielcarski M. Separation of ytterbium from ${ }^{177} \mathrm{Lu} / \mathrm{Yb}$ mixture by electrolytic reduction and amalgamation. Sop Transactions on Applied Chemistry, 2014, vol. 1, no. 2, pp. 6-13.

8. Chakravarty R., Das T., Dash A., Venkatesh M. An electro-amalgamation approach to isolate no-carrier-added ${ }^{177} \mathrm{Lu}$ from neutron irradiated Yb for biomedical applications. Nuclear Medicine and Biology, 2010, vol. 37, pp. 811-820.

9. Boldyrev P.P., Kurochkin A.V., Nurtdinov R.F., Proshin M.A., Chuvilin D.Yu., Yashin Yu.A. Electrochemical method for producing radionuclide Lu-177 with high specific activity. Moscow Univ. Chem. Bull., 2016, vol. 71, no. 3, pp. 193-198. 
10. Moeller T., Kremers H. Observations on the rare earths extraction of ytterbium from rare earth mixtures with sodium amalgam. Ind.Eng. Chem., 1945, vol. 17, no. 12, pp. 798-800.

11. Boldyrev P.P., Zagryadskii V.A., Erak D.Yu. Possibility of obtaining high-activity ${ }^{177} \mathrm{Lu}$ in the IR-8 research reactor. Atomic Energy, 2017, vol. 121, no. 3, pp. 208-213.

12. Lebedev N.A., Novgorodov A.F., Misiak R., Brockmann J., Rosch F. Radiochemical separation of no-carrier-added ${ }^{17} \mathrm{Lu}$ as produced via the ${ }^{176} \mathrm{Yb}(n, \gamma)^{177} \mathrm{Yb} \rightarrow{ }^{177} \mathrm{Lu}$ process. Applied Radiation and Isotopes, 2000, vol. 53, pp. 421-425.

13. McCoy H.N. Europium and ytterbium amalgams. J. Am. Chem. Soc., 2002, vol. 63, no. 6, pp. 1622-1624.

14. Kosynkin V.D., Molchanova T.V., Zharova E.V. Lutetium and ytterbium separation by ion-exchange chromatography. Atomic Energy, 2017, vol. 121, no. 6, pp. 443-447.

15. Bilewicz A., Zuchowska K., Bartos B. Separation of $\mathrm{Yb}$ as $\mathrm{YbSO}_{4}$ from the ${ }^{176} \mathrm{Yb}$ target for production of ${ }^{177} \mathrm{Lu}$ via the ${ }^{176} \mathrm{Yb}(\mathrm{n}, \gamma)^{177} \mathrm{Yb} \rightarrow{ }^{177} \mathrm{Lu}$ process. J. Radioanal. Nucl. Chem., 2009, vol. 280 , no. 1, pp. 167-169.
16. Dash A., Chakravarty R., Knapp F.F., Pillai A. Indirect production of no carrier added (NCA) ${ }^{177} \mathrm{Lu}$ from irradiation of enriched ${ }^{176} \mathrm{Yb}$ : options for ytterbium/lutetium separation. Current $R a$ diopharmaceuticals, 2015, vol. 8, no. 2, pp. 107-118.

17. Steeman E., Temmerman E., Verbeek F. Electrochemical reduction of the lanthanide ions. Part 1. First reduction step of ytterbium(III) in acidic $1 \mathrm{M} \mathrm{NaClO}_{4}$ solution. J. Electroanal. Chem., 1978, vol. 89, pp. 97-111.

18. Rabockai T. Electrochemical reduction of ytterbium in perchloric media. J.Electroanal. Chem., 1977, vol. 76, pp. 83-89.

19. Steeman E., Temmerman E., Verbeek F. Electrochemical reduction of the lanthanide ions. Part II. Second reduction step of europium(III), ytterbium(III) and samarium(III) in acidic tetramethylammonium perchlorate solution. J. Electroanal. Chem., 1978, vol. 89, pp. 113-122.

20. Barton G.B., Kurbatov J.D. Dependence of the diffusion current of ytterbium on supporting electrolyte and pH. J. Phys. Chem., 1949, vol. 53, no. 5, pp. 683-690.

Received: 8 May 2019.

\section{Information about the authors}

Andrey V. Korshunov, Dr. Sc., professor, National Research Tomsk Polytechnic University.

Svetlana V. Kovaleva, Dr. Sc., professor, Tomsk State Pedagogical University.

Polina V. Abramova, Cand. Sc., senior lecturer, National Research Tomsk Polytechnic University.

Dmitry A. Gorlushko, Cand. Sc., associate professor, National Research Tomsk Polytechnic University. 